Provided for non-commercial research and education use. Not for reproduction, distribution or commercial use.

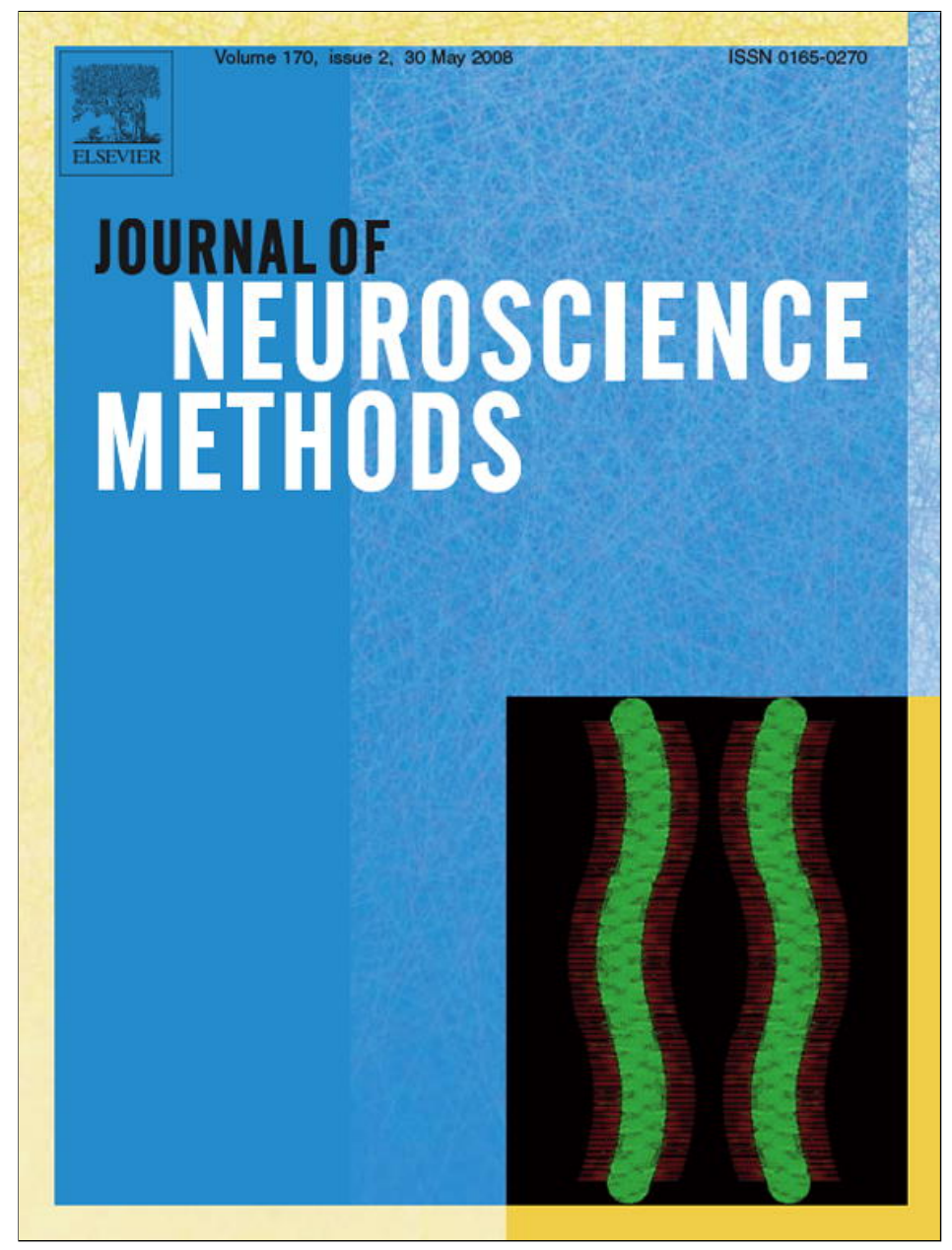

This article appeared in a journal published by Elsevier. The attached copy is furnished to the author for internal non-commercial research and education use, including for instruction at the authors institution and sharing with colleagues.

Other uses, including reproduction and distribution, or selling or licensing copies, or posting to personal, institutional or third party websites are prohibited.

In most cases authors are permitted to post their version of the article (e.g. in Word or Tex form) to their personal website or institutional repository. Authors requiring further information regarding Elsevier's archiving and manuscript policies are encouraged to visit:

http://www.elsevier.com/copyright 


\title{
Granger causality: Cortico-thalamic interdependencies during absence seizures in WAG/Rij rats
}

\author{
Evgenia Sitnikova $^{\mathrm{a}, *}$, Taras Dikanev ${ }^{\mathrm{b}, \mathrm{c}, 1}$, Dmitry Smirnov ${ }^{\mathrm{c}, 2}$, \\ Boris Bezruchko $^{\text {b,c,3 }}$, Gilles van Luijtelaar ${ }^{\mathrm{d}, 4}$ \\ a Department of Neuroontogenesis, Institute of Higher Nervous Activity and Neurophysiology, Russian Academy of Sciences, \\ Butlerova Str. 5A, 117485 Moscow, Russia \\ b Saratov State University, Department of Nano- and Biomedical Technologies, Astrakhanskaya Str. 83, 410012 Saratov, Russia \\ ${ }^{\mathrm{c}}$ Laboratory of Nonlinear Dynamical Modeling, Saratov Branch of Institute of RadioEngineering and Electronics, \\ Russian Academy of Sciences, Zelyonaya Str. 38, 410019 Saratov, Russia \\ ${ }^{\mathrm{d}}$ Department of Biological Psychology, Radboud University Nijmegen, Nijmegen Institute of Cognition and Information, \\ PO 9104, 6500 HE Nijmegen, The Netherlands
}

Received 12 October 2007; received in revised form 14 December 2007; accepted 18 January 2008

\begin{abstract}
Linear Granger causality was used to identify the coupling strength and directionality of information transport between frontal cortex and thalamus during spontaneous absence seizures in a genetic model, the WAG/Rij rats. Electroencephalograms were recorded at the cortical surface and from the specific thalamus. Granger coupling strength was measured before, during and after the occurrence of spike-wave discharges (SWD).

Before the onset of SWD, coupling strength was low, but associations from thalamus-to-cortex were stronger than vice versa. The onset of SWD was associated with a rapid and significant increase of coupling strength in both directions. There were no changes in Granger causalities before the onset of SWD. The strength of thalamus-to-cortex coupling remained constantly high during the seizures. The strength of cortex-to-thalamus coupling gradually diminished shortly after the onset of SWD and returned to the pre-SWD level when SWD stopped. In contrast, the strength of thalamus-to-cortex coupling remained elevated even after cessation of SWD.

The strong and sustained influence of thalamus-to-cortex may facilitate propagation and maintenance of seizure activity, while rapid reduction of cortex-to-thalamus coupling strength may prompt the cessation of SWD. However, the linear estimation of Granger coupling strength does not seem to be sufficient for predicting episodes with absence epilepsy.
\end{abstract}

(C) 2008 Elsevier B.V. All rights reserved.

Keywords: Granger causality; Functional coupling; Thalamo-cortical associations; Electroencephalogram; Spike-and-wave seizures; Thalamo-cortical network; Animal model

\section{Introduction}

Over the years electroencephalography is widely used in clinical practice for the investigation, classification and diagnosis of epileptic disorders. The electroencephalogram (EEG)

\footnotetext{
* Corresponding author. Tel.: +7 49533470 61; fax: +7 4953388500.

E-mail addresses: jenia-s@mail.ru (E. Sitnikova),tvdikanev@yandex.ru (T. Dikanev),smirnovda@yandex.ru (D.Smirnov),bbp@sgu.ru (B.Bezruchko), G.vanLuijtelaar@nici.ru.nl (G. van Luijtelaar).

1 Tel.: +7 8452 498037; fax: +7 8452272401 .

2 Tel.: +7 8452 511180; fax: +78452272401 .

3 Tel.: +7 8452 511180/498037; fax: +7 8452272401 .

4 Tel.: +31 24 3615612; fax: +31 243616066 .
}

provides valuable information in patients with typical and atypical epileptic syndromes and offers also important prognostic information.

Absence epilepsy, previously known as petit mal, is classically considered as non-convulsive generalized epilepsy (classification of the International League Against Epilepsy, ILAE) of unknown etiology (refs. in Panayiotopoulos, 1997). Clinically, absence seizures occur abrupt, last several seconds up to a minute and are accompanied by a brief decrease of consciousness that interrupts normal behavior. Absences may either have or have no facial automatisms, e.g. minimal jerks and twitches of facial muscles, and eye blinks. In humans, EEGs during typical absence seizures are characterized by the occurrence of generalized $3-5 \mathrm{~Hz}$ spike-wave complexes which have an 
abrupt on- and offset (Bosnyakova et al., 2007; Panayiotopoulos, 1997). Similar EEG paroxysms, spike-and-wave discharges (SWD) appear in rat strains with a genetic predisposition to absence epilepsy, such as GAERS (Genetic Absence Epilepsy Rats from Strasbourg; Vergnes, 1987) and WAG/Rij (Wistar Albino Glaxo from Rijswijk, Coenen and van Luijtelaar, 2003; see also http://www.socsci.ru.nl/wagrij/info.0.html). The EEG waveform and duration (1-30s, mean $5 \mathrm{~s}$ ) of SWD in rats and in humans are comparable, but the frequency of SWD in rats is higher, $8-11 \mathrm{~Hz}$ (Midzianovskaia et al., 2001; Sitnikova and van Luijtelaar, 2007; van Luijtelaar and Coenen, 1986).

Several modern computational techniques and advanced methods of EEG analysis have been developed to extract "hidden" information from the EEG in order to localize the region of onset and to anticipate the onset of 'absences' as early as possible (in rats, Meeren et al., 2002; Refs. for human data in Mormann et al., 2007). Our experiments are carried out in WAG/Rij rats (van Luijtelaar and Coenen, 1986). Every subject of this strain has typical absence seizures that are accompanied with spontaneous SWD in the EEG. Previously we used EEG coherence to measure neuronal synchrony between populations of thalamic and cortical neurons (Sitnikova and van Luijtelaar, 2006). We found that the onset of SWD was characterized by area-specific increase of coherence and supports the idea that the cortico-thalamo-cortical circuitry is primarily involved in the initiation and propagation of SWD (Meeren et al., 2005; Steriade, 2005). Coherence is a traditional measure of linear correlations between two EEG channels in the frequency domain, but it does not assume directionality of inter-channel interactions and does not provide temporal (time-domain) information of the EEG signals (Challis and Kitney, 1991). Granger causality compensates for these limitations (Ancona et al., 2004; Feldmann and Bhattacharya, 2004; Granger, 1969; Hlavackova-Schindler et al., 2007; Pereda et al., 2005). Granger causality concept can be used to determine directional coupling characteristics between recording sites in intracranial EEG and to reveal active abnormal causal relationships in epileptogenic networks (Chávez et al., 2003; Kaminski et al., 2001). Usually, Granger causality estimations are performed in long time intervals that include a dozen or even more, basic periods. The pairwise analysis is based on the construction of vector autoregressive (AR) models from bivariate data that estimates how well the current measure of one process can improve the prediction of the future of another process (Granger, 1969). Here we apply Granger causality to measure the strength of bidirectional functional interactions between neuronal assembles in thalamus and frontal cortex.

The present work aims to measure bidirectional (feedforward and feedback) network interdependences between local field potentials recorded simultaneously from the specific thalamus and the frontal cortex. Granger causality concept will be used to characterize the strength of functional connectivity between the cortical and thalamic EEGs for both directions before, during, and after SWD in rats.

\section{Materials and methods}

\subsection{Animals and EEG data acquisition}

Experiments were performed in five male 11-12-month old WAG/Rij rats. The recordings were done at the Department of Biological Psychology, Radboud University Nijmegen in accordance with the European Communities Council Directive (86/609/EEC). Experiments were approved by the Ethical Committee on Animal Experimentation of Radboud University Nijmegen. Distress and suffering of animals were minimal.

EEGs were recorded from brain areas in which seizure activity is known to be the most robust: in the frontal cortex and in the ventroposteromedial thalamic nucleus, VPM (Vergnes et al., 1987). Stainless steel EEG electrodes were implanted during stereotactic surgery under isofluorane anesthesia. One electrode was placed epidurally over the frontal cortex [AP 2; L 2.5] and the other depth electrode was implanted into the ventroposteromedial thalamic nucleus [VPM, AP - 3.5; L 2.5; $H$ 7.2]. Two additional electrodes, ground and reference, were placed symmetrically over the two hemispheres of the cerebellum. All electrodes were identical (diameter $0.25 \mathrm{~mm}$ ) and had non-insulated tips. The coordinates are given in $\mathrm{mm}$ relative to bregma according to the rat brain atlas of Paxinos and Watson (1986).

After the surgery, animals were allowed to recover during at least 10 days. During this recovery period, animals received postsurgery care and their weight was monitored. Upon completion of the EEG recording sessions, rats were deeply anesthetized with overdose of sodium pentobarbital $(200 \mathrm{mg} / \mathrm{kg}$ i.p.) and their brains were stained with Nissl. Electrode positioning was verified using the atlas of the rat brain (Paxinos and Watson, 1986).

EEG recordings were made in freely moving rats in a Faraday cage. Each recording session lasted from 5 to $7 \mathrm{~h}$ during the dark period of the day-night cycle. EEG signals were fed into a multi-channel differential amplifier, filtered between 1 and $200 \mathrm{~Hz}$, digitized with 1024 samples/second per channel (CODAS software) and stored on hard disk.

SWD appeared in EEG as a train of stereotypic repetitive $7-10 \mathrm{~Hz}$ spikes-and-waves with high amplitude (that exceeded the background more than three times); SWD lasted longer than $1 \mathrm{~s}$ (Midzianovskaia et al., 2001; van Luijtelaar and Coenen, 1986). SWD were detected automatically in the frontal EEG records using the algorithm and original software developed by Dr. Ir. P.L.C. van den Broek (NICI, Radboud University Nijmegen, the Netherlands). This method is based on the detection of steep changes in the EEG.

\subsection{Granger causality}

Let $\left\{x_{n}\right\}$ and $\left\{y_{n}\right\}$ be the two EEG signals (time series) recorded simultaneously in different brain loci. $x_{n}$ and $y_{n}$ are EEG values measured at the $n$-th time point. In order to study causal relations between $x$ and $y$, we use Granger's approach and analyze prediction improvement. 
First, we fit a univariate autoregressive (AR) model to the EEG data. The model takes the form

$x_{n}=f\left(x_{n-1}, x_{n-2}, \ldots, x_{n-d}\right)$.

It relates current EEG value to the $d$ previous values, $f$ is some function, e.g., algebraic polynomial whose order and coefficients are to be estimated from the observed data. Eq. (2) shows a widely used linear AR model

$x_{n}=\alpha_{0}+\sum_{i=1}^{d} \alpha_{i} x_{n-i}$.

Coefficients $\alpha_{i}$ are selected in order to minimize the mean squared error, $\varepsilon_{x}^{2}$

$\varepsilon_{x}^{2}=\frac{1}{N} \sum_{n}\left(x_{n}-\left(\alpha_{0}+\sum_{i=1}^{d} \alpha_{i} x_{n-1}\right)\right)$,

where $N$ is the number of predicted samples in a time series. If the number of model parameters is much less than the number of data points used for the estimation, a minimal value $\varepsilon_{x}^{2}$ can characterize the accuracy of the model: the less the error, the better the model (self-predictability of the model).

Causal relations between process $y$ and process $x$ are present when prediction of signal $\left\{x_{n}\right\}$ can be improved by incorporation into the model the past of signal $\left\{y_{n}\right\}$ (illustrated in Fig. 1A). As a result, we construct a 'joint' AR model Eq. (4)

$x_{n}=f\left(x_{n-1}, \ldots, x_{n-d_{1}}\right)+g\left(y_{n-1}, \ldots, y_{n-d_{2}}\right)$,

where $f$ and $g$ are polynomials that we determine from the current data. Function $f$ is the same as in the individual model Eq. (1) while function $g$ describes the influence of process $y$ on process $x$. Number $d_{1}$ is the same as the dimension $d$ of the individual model Eq. (1). The number $d_{2}$ describes 'inertial' properties of the influence. If $d_{2}=1$, then the influence is instantaneous, otherwise it is non-local in time. Different values of $d_{1}$ and $d_{2}$ $\in(1 ; 25)$ are tested in order to select those values that provide the most faithful results. In the present study we used linear AR models Eq. (5):

$x_{n}=\alpha_{0}+\sum_{i=1}^{d_{1}} \alpha_{i} x_{n-i}+\sum_{i=1}^{d_{2}} \beta_{i} y_{n-i}$.

In Eq. (5) coefficients $\alpha_{i}$ are chosen using the least-squares estimations method. This method examines mean squared prediction error, $\varepsilon_{x y}^{2}$, and when this error appears to be less then the $\varepsilon_{x}^{2}$, it is assumed that process $y$ influences process $x$ (Fig. 1A). In order to measure coupling between channels, we use the relative prediction improvement, the so-called Granger-Sargent statistic (Hlavackova-Schindler et al., 2007):

$s_{x y}^{2}=\frac{\varepsilon_{x}^{2}-\varepsilon_{x y}^{2}}{\varepsilon_{x y}^{2}}$.

Thus, the influence of channel $\left\{y_{n}\right\}$ on channel $\left\{x_{n}\right\}$ is characterized by the value of the normalized prediction improvement $s_{x y}^{2}$ and the reverse influence of $\left\{x_{n}\right\}$ on $\left\{y_{n}\right\}, s_{y x}^{2}$ is described
EEG channel $\left\{x_{n}\right\}$ (the thalamus)

(A) $100 \mu \mathrm{V}$
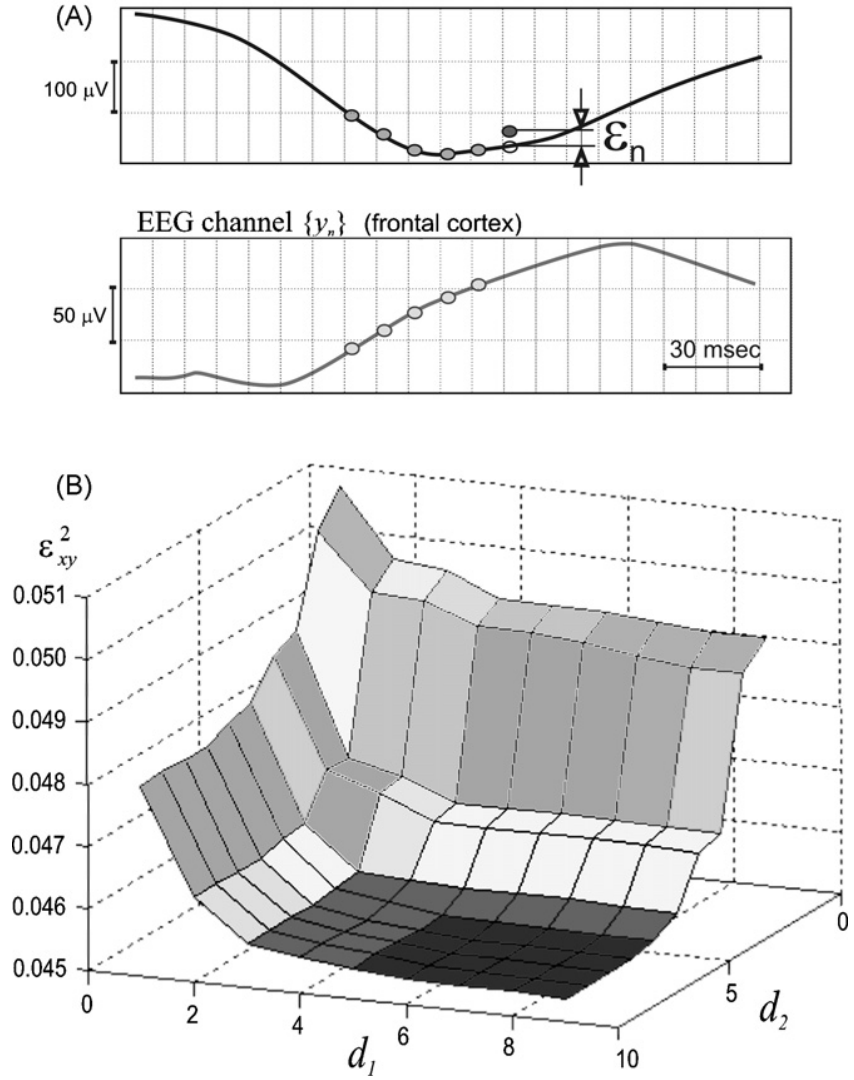

Fig. 1. Estimation of Granger causality between EEG signals as recorded at thalamus and frontal cortex. The autoregressive model (AR) is used to characterize amplitude changes in two EEG signals, $\left\{x_{n}\right\}$ and $\left\{y_{n}\right\}$ over time. (A) In the joint AR model, the information about the past of EEG signal $\left\{y_{n}\right\}$ improves the prediction of EEG signal $\left\{x_{\mathrm{n}}\right\}$ (see Eq. (4)) with a reduction of the instantaneous prediction error, $\varepsilon_{n}$. The squared errors, $\varepsilon_{x y}^{2}$, characterize the quality of prediction of the chosen AR model. The value of $\varepsilon_{x y}^{2}$ depends on the number of samples in signals $\left\{x_{n}\right\}$ and $\left\{y_{n}\right\}$ used in the AR model, so-called model dimensions, $d$. (B) Dependencies between the prediction errors, $\varepsilon_{x y}^{2}$, and the model dimensions in our linear AR that predicted the future of the signal $\left\{y_{n}\right\}\left(d_{1}\right.$ and $d_{2}$ is the number of samples in the signal $\left\{x_{n}\right\}$ and signal $\left\{y_{n}\right\}$ correspondingly). An increase of dimensions $d_{1}$ and $d_{2}$ resulted in a decrease of the least mean squared error and the quality of predictions was improved until $d_{1}=d_{2}=5$. Further increase of dimensions did not improve the accuracy of prediction, therefore in our computations we used $d_{1}=d_{2}=5$.

by an equation similar to Eq. (6) in which $x$ and $y$ should be interchanged.

\subsection{Application of Granger causality to EEG data}

Nonlinear dynamics techniques consider EEG signals as nonlinear process and try to extract some nonlinear features from it. Typically, they require long epochs of stationary data (e.g., Arnhold et al., 1999; Le Van Quyen et al., 1999; Schiff et al., 1996). Estimations of Granger causality, either linear or nonlinear, are performed under the same conditions. Linear estimates are usually less sensitive to the epoch length, because they are based on relatively simple models (with fewer free parameters). However, the EEG is known to be highly non-stationary (Kaplan, 1998). This should be taken into account in order to specify the 
proper time window length for the estimation of autoregressive models when estimating Granger causality.

\subsubsection{The choice of length of moving window}

In fact, non-stationary is an intrinsic feature of the EEG signal that accounts for complex dynamics of electrical brain activity (Dikanev et al., 2005). However, the classical estimation of Granger causality requires stationary data. Therefore we segmented the EEG into relatively short epochs in which the EEG signal reveals quasi-stationary behavior.

In order to get a correct approximation to the non-stationary EEG data with the AR model, it is important to define the optimal size of the moving time-window. By shortening the time-window, it is possible to improve the time resolution, but this reduces the reliability of the AR models. In non-stationary EEG data, the time-window should include several repetitive elements. In our case, the time-window lasts $0.5 \mathrm{~s}$; this corresponds to four spike-wave cycles. This size of the time-window was found to be optimal. If the estimation window was shorter than $0.5 \mathrm{~s}$, Granger causality estimations were unstable and longer time-window (up to $1 \mathrm{~s}$ ) did not improve the stability of coupling estimates.

\subsubsection{Selection of the polynomial order \\ (linearity-nonlinearity in EEG signals)}

Originally, Granger causality principles were formulated without any assumption about the linearity or nonlinearity of the systems. Traditional Granger causality measures were based on linear models (Granger, 1969; Granger and Newbold, 1977). In order to make a choice between linear and nonlinear AR models, we compared prediction accuracy of these two models. It was found that the introduction of nonlinearity (such as polynomials of the second and third order) had no significant influence on the prediction quality of the AR model. It was additionally found that a linear AR model was sufficient to describe the dynamic behavior of the baseline EEG. It suggests a predominance of the linear causal relations in non-seizure EEG. In contrast, seizure activity (SWD) contained a nonlinear component, which was not yet modeled. However, the construction of a specific nonlinear AR model that describes seizure-related processes in the EEG is beyond the scope of the present paper.

\subsubsection{Adjusting parameters of AR model (the choice of 'dimensions')}

The linear AR models Eqs. (2) and (5) are used to calculate the coupling characteristics $s_{x y}^{2}$ and $s_{y x}^{2}$. Fig. 1B shows the typical dependence of prediction error $\varepsilon_{x y}^{2}$ on the dimensions $d_{1}$ and $d_{2}$ of the AR model for a $0.5 \mathrm{~s}$ time-interval of SWD. By increasing the dimensions of the model $d_{1}$ and $d_{2}$ from 1 to 5 , the error of prediction decreases and a minimal error was obtained when both $d$-values were equal to 5 . Further increase of $d_{1}$ and $d_{2}$ did not improve the accuracy of predictions, therefore, $d_{1}=d_{2}=5$ were selected as optimal dimensions for the chosen model. Such a dependence is typical for the prediction error $\varepsilon^{2}$ as well.

All this suggests that also in our linear AR model the past of one signal improves the prediction of the other signal. This model provided significant and stable predictions with the chosen parameters (dimensions and time-window size), therefore, it was an appropriate model for the analysis of pairwise causal relations in the chosen EEG pairs.

\subsubsection{Statistical evaluation of causal interactions}

Coefficients of Granger causality (prediction improvements) were computed using EEG data from the cortical surface (frontal cortex) and from the specific part of the thalamus (ventral basal complex). The first and the last spike in spike-wave sequences were used to mark the onset and the offset of seizure activity. Statistical analysis of the thalamus-to-cortex $\left(s_{x y}^{2}\right)$ and cortexto-thalamus $\left(s_{y x}^{2}\right)$ causalities was performed in two 10-s EEG epochs (Fig. 2A). The first epoch included two $5 \mathrm{~s}$ successive intervals, one before seizure onset (pre-SWD), the second was the first $5 \mathrm{~s}$ of a seizure (SWD-start). The second 10-s epoch included the last $5 \mathrm{~s}$ of a SWD (SWD-end) and the first $5 \mathrm{~s}$ after a seizure (post-SWD). Coefficients of Granger causality were computed with bin $=0.0049$ ( 5 samples/1024) and averaged per $0.2 \mathrm{~s}$ and per rat. Factorial ANOVA (repeated measures) and post hoc tests (LSD and $t$-tests for paired observations) were used for the statistical analysis.

\subsubsection{Evaluation of statistical significance with surrogate test estimation}

There are some undesirable factors (EEG noise, nonlinearities, etc.) that can influence Granger causality measures and might mislead analysis. In order to control for these unwanted factors and evaluate the statistical significance of the Granger causality parameters, we performed surrogate data tests. Surrogate signals were constructed by taking two apparently uncoupled EEG signals recorded in the cortex and thalamus. For that purpose, two randomly chosen SWD were selected in the cortex and thalamus. Either the onset or the end of SWD were matched. Surrogate tests were performed using EEG data from all animals. In total, 1000 random pairs of SWD were made to construct an ensemble of surrogate time series which modelled uncoupled process in the thalamo-cortical system. Granger causalities in surrogate and in original data were computed using the same algorithm. The surrogate Granger measures in the uncoupled pairs were analyzed statistically by computing $95 \%$-percentiles of their distributions, $s_{x y, 0.95}^{2}$ and $s_{y x, 0.95}^{2}$, respectively. The results of surrogate tests, $s_{x y, 0.95}^{2}$ and $s_{y x, 0.95}^{2}$, were plotted in Fig. 2B and $\mathrm{C}$ against the true data values, $s_{x y}^{2}$ and $s_{y x}^{2}$. Interdependence in EEG pair can be regarded as significant (at the significance level $p=0.05$ ) whenever the true values $s_{x y}^{2}$ and $s_{y x}^{2}$ appeared above $95 \%$-percentiles of the corresponding surrogate values $s_{x y, 0.95}^{2}$ and $s_{y x, 0.95}^{2}$. This surrogate test confirmed that interdependencies between EEG signals were significant in both directions.

\section{Results}

All SWD were detected automatically in the full length EEG recordings (6-7 h). In total, 53, 111, 34, 33 and 63 epileptic discharges in five rats were detected and analyzed. 
(A)
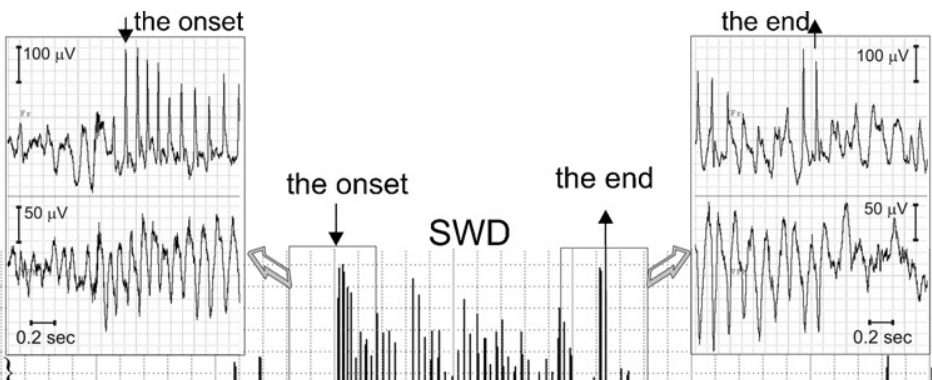

Frontal cortex $\left\{y_{n}\right\}$
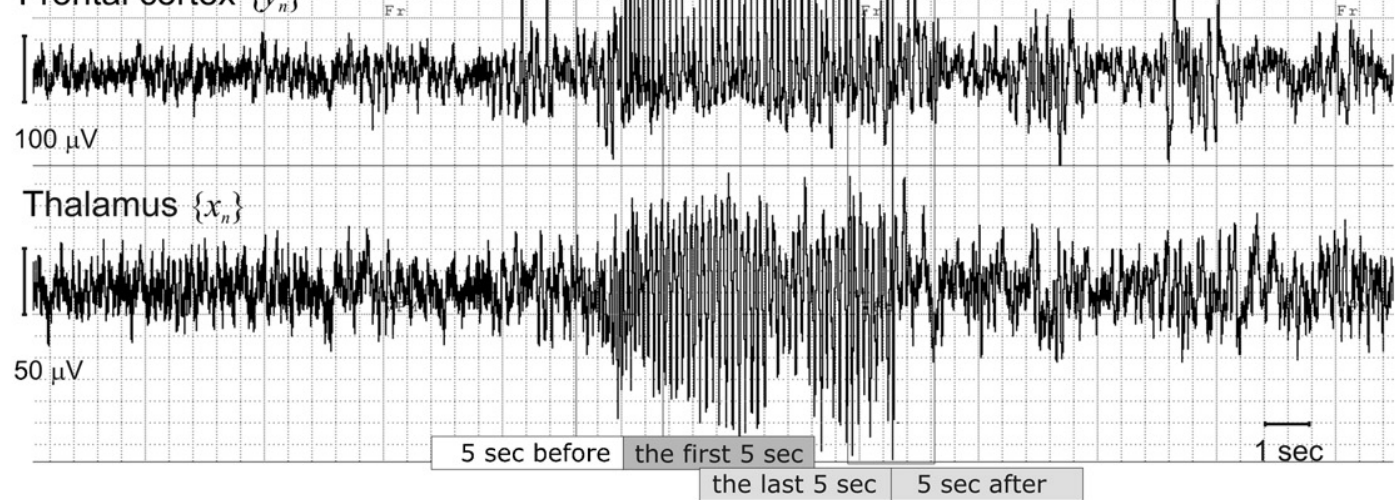

(B)

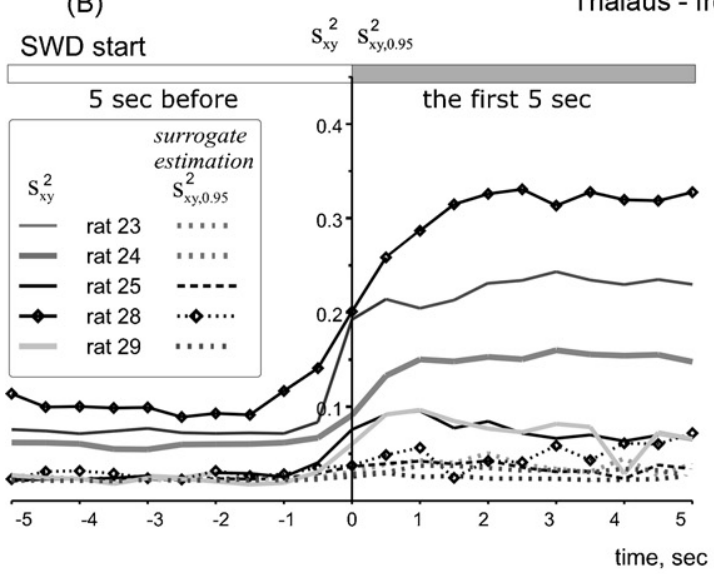

Thalaus - frontal cortex

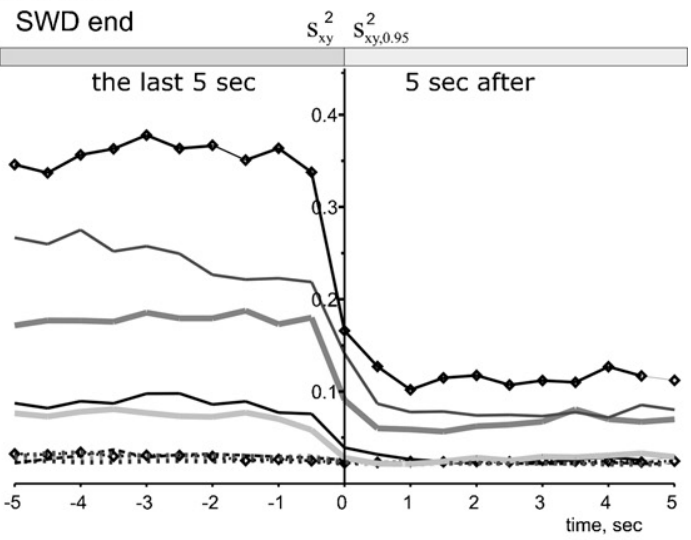

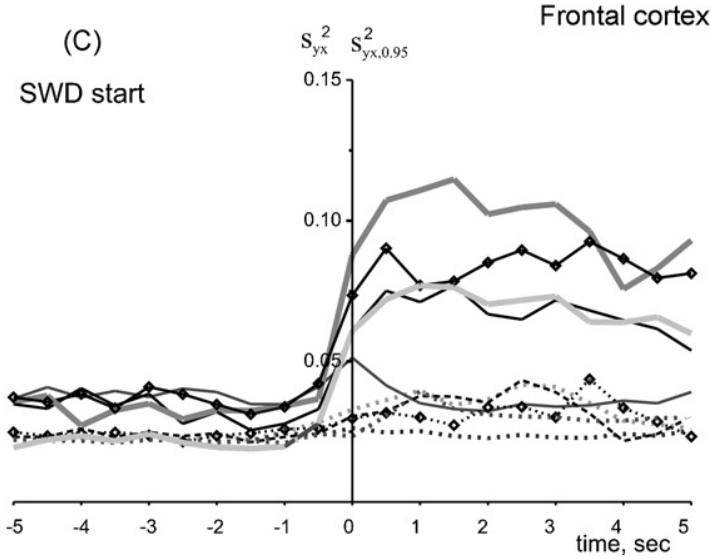

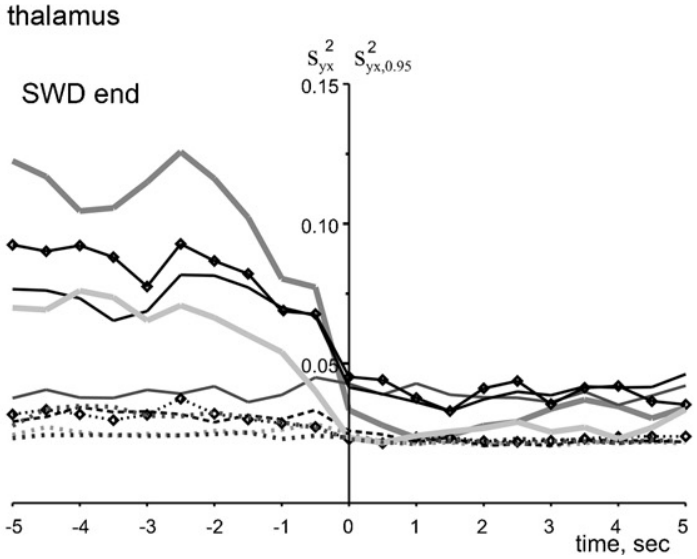

Fig. 2. Application of Granger causality in WAG/Rij rat model of absence epilepsy. (A) Electroencephalographic records of spike-wave discharges (SWD) in the frontal cortex and in the specific ventroposteromedial thalamic nucleus. Coefficients of Granger causality were computed in two 10-s epochs of continuous data $(5+5 \mathrm{~s})$ including pre-SWD/SWD and SWD/post-SWD (indicated by horizontal arrows). (B, C) The presence of SWD was associated with significant (and reversible) changes in Granger causality in both directions. Surrogate data tests (dotted lines) were performed in each animal in order to validate the results of Granger causality estimations. Surrogate values were very small and did not reveal any dynamic changes, suggesting that seizures affected the coupling strength in both directions. Note the large individual variations and two-hold difference in absolute values of cortex-to-thalamus and thalamus-to-cortex causalities. 


\subsection{Dynamics of cortico-thalamo-cortical casual interactions at the on- and offset of epileptic discharges}

Fig. 2B shows the dynamics of Granger causality during absence seizures. Before the onset of SWD, the Granger causality was weak and remained constant until SWD began. The first SWD-related disturbances of Granger's casual relationships were observed about half a second before SWD-onset. This effect was provoked by the seizure itself because the $0.5 \mathrm{~s}$ timewindow started to include or capture seizure activity. No changes in Granger causalities were found earlier than $0.5 \mathrm{~s}$ before SWD onset, suggesting that a linear approximation does not seem to be suitable for prediction of absence seizures. It can be concluded that the linear cortico-thalamo-cortical associations are reinforced during SWD.

It is important that in all rats surrogate values $s_{x y, 0.95}^{2}$ and $s_{y x, 0.95}^{2}$ were almost constant in time, equal in both directions and were much smaller (around 0.02-0.04) than the true data estimates, $s_{x y}^{2}$ and $s_{y x}^{2}$ (Fig. 2B and C). The difference between the true $\left(s_{x y}^{2}\right.$ and $\left.s_{y x}^{2}\right)$ and surrogate $\left(s_{x y, 0.95}^{2}\right.$ and $\left.s_{y x, 0.95}^{2}\right)$ values strengthened our outcomes and confirmed that mutual interdependencies between cortex and thalamus during SWD was statistically significant.

The immediate onset of SWD was associated with a rapid growth of causal relations ( $s_{x y}^{2}$ and $s_{y x}^{2}$, Fig. 2B, C and Fig. 3A). Granger causality reached its maximum within half a second after seizure onset (at that moment the time-window completely shifted from pre-SWD to SWD) and remained high during the first $5 \mathrm{~s}$ of a seizure.

Quantitative data in Table 1 shows that the onset of SWD was characterized by a significant increase of causalities in both directions as compared to pre-SWD $(F=20.53$, d.f. $=1.4$, $p<0.02)$ and that the ascending influence thalamus-to-cortex tended to be stronger than the descending influence cortexto-thalamus $(F=4.75$, d.f. $=1.4, p<0.1)$ (Fig. 3B; Table 1). Interestingly, the occurrence of SWD was associated with a tendency for a larger increase of the thalamus-to-cortex as compared to the cortex-to-thalamus coupling $\left(\Delta s_{x y}^{2}\right.$ (SWD- start $)=0.117$ versus $\Delta s_{y x}^{2}($ SWD-start $)=0.037, F($ time $)=2.43$, d.f. $=1.4, p<0.1)$. All this suggests that a reinforcement of pre-SWD existing predominant thalamus-to-cortex coupling accompanied the occurrence of SWD.

\subsection{Gross changes of Granger causality in the cortico-thalamo-cortical system before, during and after absence seizures}

In spite of large between-subject variability in the values of Granger causality causalities (individual data are shown in Fig. 2B and C), a similar trend of SWD-related changes in Granger causality was observed in all subjects. Group statistics in Fig. 3 illustrate that the mutual relationships between the specific ventroposteromedial nucleus of the thalamus and the frontal cortex became stronger after the onset of SWD. The offset of SWD was characterized by a slight and gradual decrease of Granger causalities in both directions.

A two-factor ANOVA was used to compare the average values of coupling strength on different stages of SWD. Factor 'time' had four levels: pre-SWD, SWD-start, SWD-end, post-SWD and factor 'direction' had two levels: cortex-to-thalamus and thalamus-to-cortex. Both factors were significant: $F($ time $)=58.4$, d.f. $=3.992, p<0.0001$ and $F$ (direction $)=319.6$, d.f. $=1.992, p<0.0001$, as well as the interaction $F$ $($ time $\times$ direction $)=17.9$, d.f. $=3.992, p<0.0001$. Post hoc LSD tests revealed significant difference of causalities in both directions at pre-SWD and during SWD (Fig. 3B). The strength of cortex-to-thalamus coupling before (pre-SWD) and after (postSWD) did not differ (Fig. 3B).

In contrast, the thalamus-to-cortex coupling did not return to the pre-seizure level immediately after the cessation of the epileptic electroencephalographic activity, as did the cortex-tothalamus coupling. Altogether, the analysis of Granger causality provided new information about neuronal network connectivity during absence seizures. We consider this method as a good alternative to the traditional measurements of functional interactions in the brain.

Table 1

Mean values of Granger causality as measured in $5 \mathrm{~s}$ intervals immediately before and after the onset of SWD

\begin{tabular}{|c|c|c|c|c|c|c|}
\hline & Before SWD & SWD-start (the first $5 \mathrm{~s}$ ) & $\Delta S^{2}$ (SWD-start) & SWD-end (the last $5 \mathrm{~s}$ ) & Post-SWD & $\Delta S^{2}($ SWD-end $)$ \\
\hline$S_{x y}^{2}$ & \multicolumn{6}{|c|}{ Thalamo-Cortical Coupling } \\
\hline 23 & $0.074 \pm 0.039$ & $0.177 \pm 0.093$ & 0.103 & $0.097 \pm 0.062$ & $0.045 \pm 0.028$ & -0.052 \\
\hline 24 & $0.094 \pm 0.060$ & $0.258 \pm 0.086$ & 0.164 & $0.273 \pm 0.081$ & $0.221 \pm 0.095$ & -0.052 \\
\hline 25 & $0.034 \pm 0.021$ & $0.080 \pm 0.044$ & 0.046 & $0.043 \pm 0.028$ & $0.037 \pm 0.024$ & -0.006 \\
\hline 28 & $0.109 \pm 0.060$ & $0.326 \pm 0.114$ & 0.217 & $0.341 \pm 0.117$ & $0.204 \pm 0.132$ & -0.137 \\
\hline 29 & $0.027 \pm 0.021$ & $0.080 \pm 0.044$ & 0.053 & $0.081 \pm 0.048$ & $0.079 \pm 0.041$ & -0.002 \\
\hline Total & $0.066 \pm 0.053$ & $0.183 \pm 0.125$ & $0.117 \pm 0.073^{*}$ & $0.167 \pm 0.113$ & $0.117 \pm 0.114$ & $-0.049 \pm 0.075$ \\
\hline$S_{y x}^{2}$ & \multicolumn{6}{|c|}{ Cortico-Thalamic Coupling } \\
\hline 23 & $0.060 \pm 0.033$ & $0.103 \pm 0.041$ & 0.043 & $0.064 \pm 0.036$ & $0.027 \pm 0.020$ & -0.037 \\
\hline 24 & $0.046 \pm 0.031$ & $0.054 \pm 0.032$ & 0.008 & $0.049 \pm 0.025$ & $0.058 \pm 0.035$ & 0.009 \\
\hline 25 & $0.038 \pm 0.021$ & $0.076 \pm 0.032$ & 0.038 & $0.051 \pm 0.030$ & $0.045 \pm 0.028$ & -0.006 \\
\hline 28 & $0.042 \pm 0.030$ & $0.099 \pm 0.037$ & 0.075 & $0.091 \pm 0.033$ & $0.059 \pm 0.037$ & -0.032 \\
\hline 29 & $0.022 \pm 0.018$ & $0.064 \pm 0.027$ & 0.042 & $0.054 \pm 0.026$ & $0.062 \pm 0.028$ & 0.008 \\
\hline Total & $0.042 \pm 0.030$ & $0.079 \pm 0.039$ & $0.037 \pm 0.020$ & $0.062 \pm 0.035$ & $0.047 \pm 0.032$ & $-0.012 \pm 0.024$ \\
\hline
\end{tabular}



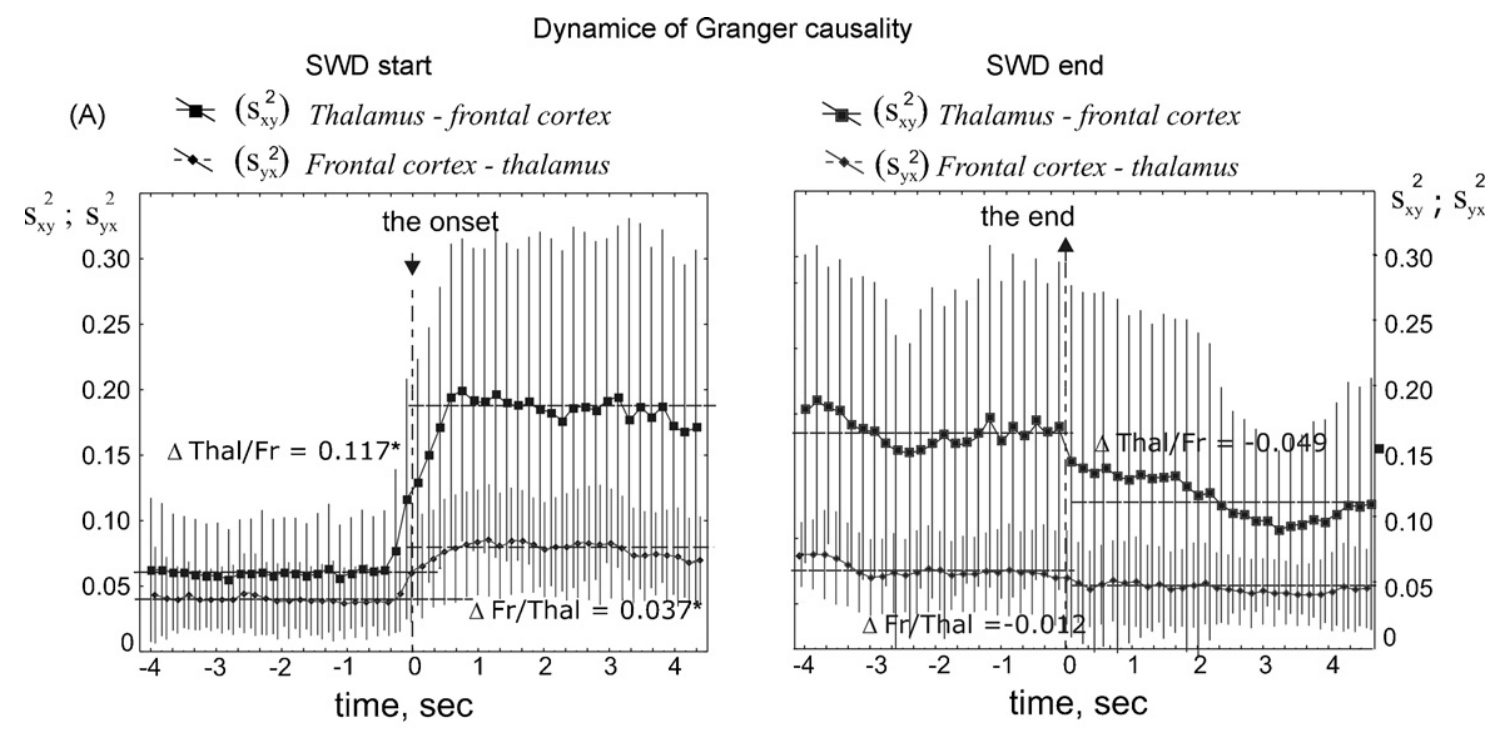

(B)

ANOVA test
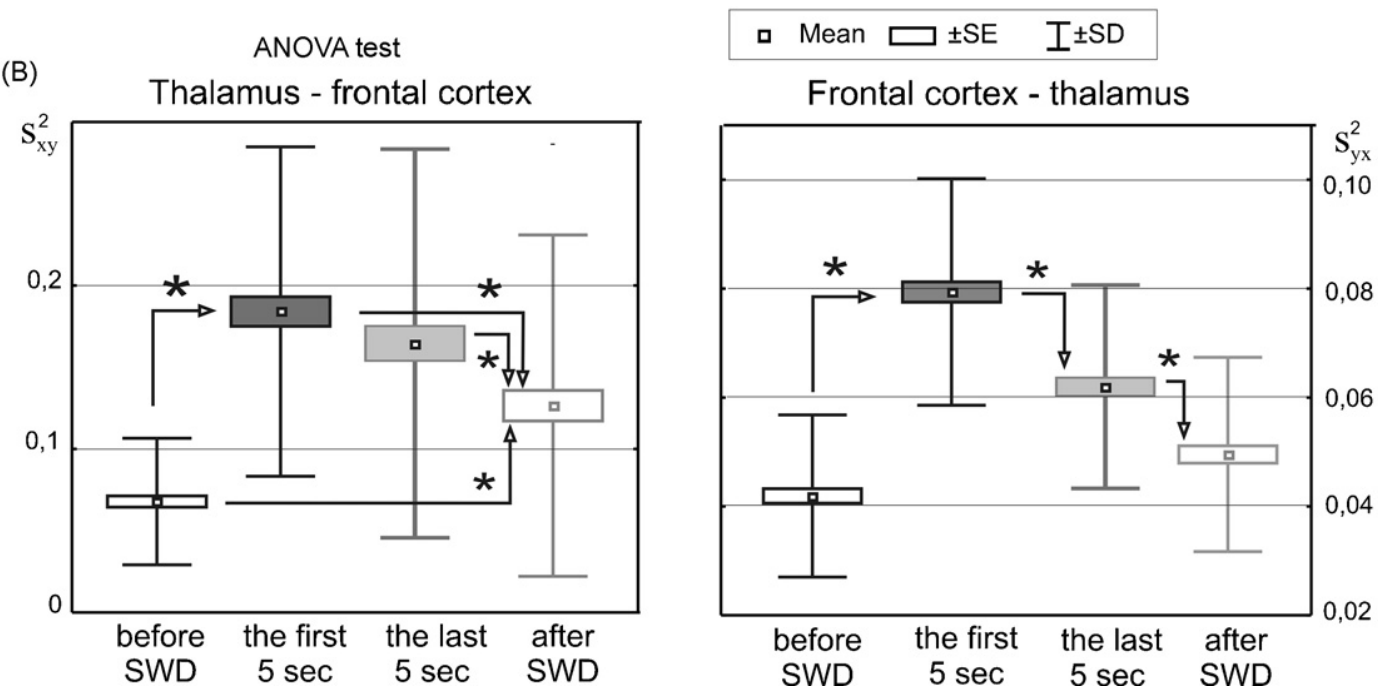

Fig. 3. Statistical assessment of changes in Granger causalities associated with the onset and end of spike-wave discharges (SWD). (A) Bidirectional causal relationship between the frontal cortex and the thalamus at the onset and the end of SWD. Coefficients of Granger causality were averaged per $0.2 \mathrm{~s}$ intervals and per rat (mean \pm S.D.). The increase in Granger causalities at the onset of SWD was abrupt and significant (ANOVA, $p<.001$ post hoc LCD-test), but seizure offset was characterized by smooth and prolonged changes in Granger causalities. (B) Group statistics of Granger causality coefficients averaged in $5 \mathrm{~s}$ intervals (five rats, 30 SWD per rat). Asterisks show significant differences (the post hoc LSD test).

\section{Discussion}

This study tackles a challenging problem of predictability of absence seizures in EEG. Using the concept of Granger causality, we measured bidirectional (straightforward and backward) linear interdependences between the thalamus and the cortex during absence seizures and obtained new yet comprehensive information about functional thalamo-cortical interactions during absence epilepsy. Traditional methods, such as crosscorrelation analysis of unit activity in a model of generalized epilepsy in cats (e.g. Steriade and Amzica, 1994) and coherence analysis in a genetic absence model (Sitnikova and van Luijtelaar, 2006) demonstrated that the genesis of generalized spike-and-wave discharges required mutual interrelationship between thalamus and cortex. The current study aims to evaluate a novel method for assessing directionality in thalamo-cortical network associations during absence epilepsy and this led to principally new conclusions. (1) Information transfer in the direction 'thalamus $\rightarrow$ frontal cortex' was more intensive than in the backward direction. This is the first indication of anisotropy in thalamo-cortical interactions (discussed in Section 4.1). (2) Coupling strength 'frontal cortex $\rightarrow$ thalamus' slightly (but significantly) increased at the onset of SWD and rapidly restored to the initial level before cessation of a seizure. A strong and sustained increase in 'thalamus $\rightarrow$ frontal cortex' interactions was found not only during SWD, but also after the end of the seizure (discussed in Section 4.2).

\subsection{Implications of the linear Granger causality in EEG analysis of absence epilepsy}

In patients with absence epilepsy, as well as in WAG/Rij rats, spike-wave seizures appear unpredictably from a normal EEG background and associated with sudden behavioral arrest, e.g. 
'absences' (Panayiotopoulos, 1997; van Luijtelaar and Coenen, 1986). As known, SWD are produced in the cortico-thalamocortical oscillatory network (Avanzini and Franceschetti, 2003; Blumenfeld, 2002; Meeren et al., 2002, 2005; van Luijtelaar and Sitnikova, 2006). Traditionally, coherence analysis is used to estimate functional associations between different brain areas (Challis and Kitney, 1991; Pereda et al., 2005). Previously, we have used coherence to measure linear thalamocortical network associations in the frequency domain (Sitnikova and van Luijtelaar, 2006). Granger causality is a time domain measure of functional interactions, assuming directionality and information transfer. Directionality of thalamo-cortical interactions during $\mathrm{SWD}$ in WAG/Rij rats has already been explored by means of nonlinear association EEG analysis (Meeren et al., 2002). It was found that directionally of thalamo-cortical coupling varied throughout the seizure and it was the most constant during the first half a second, when the cortical epileptic focus consistently led the thalamus.

Hereby, by measuring Granger causality we also planned to identify early changes in thalamo-cortical relationships that may anticipate the onset of absence seizures. We adjusted a linear autoregressive model of Granger causality in order to describe causal relations between cortical and thalamic electrical activity during absence seizures in WAG/Rij rats. Surrogate data test confirmed the statistical significance of the observed interdependence.

We first found that the linear estimation of Granger causality provided a good approximation to baseline EEG (pre-SWD), e.g. linear autoregressive model was sufficient to obtain stable results of non-seizure activity. However, with linear estimations of Granger causality we failed to identify early changes of causal relationships that may anticipate the onset of absence seizures. It is however possible that early changes of interdependencies can be described with additional nonlinear autoregressive models or with phase-synchronization methods (Le Van Quyen and Bragin, 2007). On the one hand, introduction of nonlinearity into the model may be necessary to get comprehensive information about network associations that prerequisite seizure activity or/and take place during a seizure. On the other hand, application of nonlinear AR model requires more careful selection of model parameters (such as dimensions and nonlinear model functions). This piece of work will be done in the future.

Several conclusions can be drawn from the present results. First, 'thalamus $\rightarrow$ frontal cortex' coupling characteristic, numerically, was greater than that in the opposite direction. This was found before, during and after SWD. The onset of SWD was associated with an amplification of pre-SWD existing tendencies. However, it is difficult to compare couplings in both directions to each other since thalamus and cortex signals are essentially different from each other even in respect of their waveforms (Sitnikova and van Luijtelaar, 2007). More meaningful is to trace changes in coupling characteristics over time.

Second, 'thalamus $\rightarrow$ frontal cortex' coupling remained constantly high during a seizure and did not return to pre-SWD level even after cessation of SWD. It seems intriguing that although SWD were stopped, the thalamo-cortical network did not rapidly return to the non-epileptic state and causal relationships remained abnormal. Clinically, both start and end of SWD are regarded as abrupt and unpredictable, but we observe that changes in Granger causalities at the onset of SWD were more sharp and fast as compared with that at the end of SWD (post-SWD periods were characterized by smooth and prolonged changes in Granger causalities).

Third, Granger coupling strength increased with seizure onset, although differentially in two directions: reinforcement of 'thalamus $\rightarrow$ frontal cortex' coupling was greater than that in backward direction. However, this latter comparison should be interpreted with care, as mentioned above.

\subsection{Granger causality and functional interactions in epileptic networks}

In the present study we elaborate interactions between the frontal cortex and the thalamus during absence seizures. In our rats, EEG records were made in the areas in which seizure activity is known to be the most robust, e.g. in the frontal cortex and specific thalamus (Vergnes et al., 1987). Direct anatomic connections between these areas are nearly absent, but these thalamic areas send and receive terminals to the somatosensory cortical (Jones, 1985). This midpoint, the peri-oral region of the somatosensory cortex in WAG/Rij rats, is known as 'epileptic focus' which initiate SWD (Meeren et al., 2002). In our animals, the frontal EEG electrode was relatively far away from the 'epileptic focus' and we did not measure electrical activity in focal epileptic zone. Interestingly, a French group has recently confirmed and extended the Meeren et al. (2002) data in GAERS (Polack et al., 2007). They showed that neurons in deep layers of the somatosenory cortex started firing much earlier than the first changes of local field potential could be visualized at the onset of SWD. This exaggerated neuronal firing at the early stages of SWD was only found in neurons localized in the epileptic cortical area, but it neither was detected in other areas in epileptic rats, nor in the similar areas in non-epileptic rats. Equally important is that our previous studies in WAG/Rij rats and others clearly demonstrated that neuronal activity in cortical regions outside the peri-oral area of the somatosensory cortex did not lead thalamic activity during SWD (Inoue et al., 1993; Seidenbecher et al., 1998).

We study interdependencies between two indirectly connected structures that communicate via the 'epileptic focus': 'thalamus $\leftrightarrow$ somatosensory cortex (epileptic focus) $\leftrightarrow$ frontal cortex' (Fig. 4). In order to interpret our results, we put together several theoretical considerations: the 'cortical focus' theory (Meeren et al., 2002, 2005), our concept on global and local synchronization in oscillatory networks (Sitnikova and van Luijtelaar, 2006; van Luijtelaar and Sitnikova, 2006) and ideas of 'driving' and 'modulating' connections in neuronal networks (Crick and Koch, 1998). We propose that the 'epileptic focus' not merely triggers, but also acts as a distributor of epileptic activity. In particular, seizure may easily propagate to those areas which have dense connections with the 'epileptic focus' (Sitnikova and van Luijtelaar, 2006). In order to explain the quantitative differences between coupling strength between the 


\section{Bidirectional causal relations in the thalamo-cortical network}

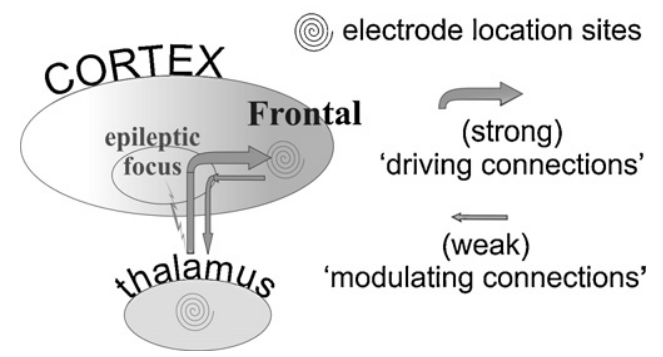

Fig. 4. Physiological implication of Granger causality estimations. Ascending and descending anatomical connections from the thalamus to the frontal cortex go through the somatosensory cortex (Jones, 1985). Somatosensory cortex in WAG/Rij rats contains the 'epileptic focus' that triggers epileptic activity (Meeren et al., 2002). As known, SWD easily spread from the epileptic somatosensory area to the frontal cortex (EEG coherence study, Sitnikova and van Luijtelaar, 2006). Also anatomic connections 'somatosensory cortex (epileptic focus) $\rightarrow$ frontal cortex' are stronger than the backward 'frontal cortex $\rightarrow$ somatosensory cortex' (Kolb, 1990), this may prompt anterior propagation of SWD from the epileptic cortical zone to the frontal cortex. The 'epileptic focus' has strong connections ('driving connections' in terms of Crick and Koch, 1998) with the frontal cortex ('somatosensory cortex (epileptic focus) $\rightarrow$ frontal cortex'), but less strong 'modulating connections' with the thalamus ('somatosensory cortex (epileptic focus) $\rightarrow$ thalamus').

thalamus and frontal cortex, we use a concept of two different kinds of network associations, e.g. 'driving' and 'modulating' connections (Crick and Koch, 1998) (Fig. 4). We hypothesize that the strong ascending coupling 'thalamus $\rightarrow$ frontal cortex' may prompt propagation and maintenance of SWD. These are 'driving connections' which are strong and induce an increase of firing activity in corresponding neurons. Less strong 'frontal cortex $\rightarrow$ thalamus' coupling may correspond to relatively weak 'modulating' connections which prevent spreading of SWD and are, therefore, involved in stopping SWD. In our case, the thalamus may excite the pathway from somatosensory to frontal cortex and this excitation may be a 'driving' force of seizure activity (Fig. 4). This schema agrees with outcomes of our coherence analysis where we found an enhancement of linear associations between somatosensory and frontal cortical areas at the onset of SWD (Sitnikova and van Luijtelaar, 2006).

\subsection{EEG studies in animals: what can we gain from them?}

Animals EEG research in epilepsy and other neurological diseases have some advantages. It was advantageous to use the WAG/Rij rat strain in the context of the present study. First, large amount of EEG epochs with seizure activity were required for the statistical analysis. These long time recordings with many SWD are not readily available in humans. Second, our aim was to clarify the role of the thalamus in absence epilepsy (this problem is still far from being solved, e.g. Avanzini and Franceschetti, 2003; Blumenfeld, 2002; Huguenard and McCormick, 2007; Meeren et al., 2005; Steriade, 2005). For this purpose our animals were implanted with depth thalamic electrodes, yet depth EEG recordings are nearly impossible in patients with absence epilepsy in which invasive depth EEG examination is exceptional. Third, human scalp EEG is affected by volume conduction (the outer tissues of the scalp act as a low pass filter). EEG electrodes in our animals were placed epidurally on the cortical surface. It is crucial that both cortical and thalamic electrodes recorded signals originating directly from the neuronal sources and the influence of volume conduction was excluded.

\section{Acknowledgements}

We gratefully acknowledge the valuable help of Prof. G.D. Kuznetsova, Prof. A. Coenen and Dr. C. van Rijn. The technical assistance of H. Krijnen, S. Hermeling, E. Willems-van Bree, J. Dederen, G. van Oyen and Dr. ir. P.L.C. van den Broek is highly appreciated.

The work was supported by the RFBR (grant no. 07-0200747) and the "Russian Science Support Foundation" (grant to Evgenia Sitnikova).

\section{References}

Ancona N, Marinazzo D, Stramaglia S. Radial basis function approach to nonlinear Granger causality of time series. Phys Rev E 2004;70:056221.

Arnhold J, Lehnertz K, Grassberger P, Elger CE. A robust method for detecting interdependences: application to intracranially recorded EEG. Physica D 1999;134:419-30.

Avanzini G, Franceschetti S. Cellular biology of epileptogenesis. Lancet Neurology 2003;2:33-42.

Blumenfeld H. The thalamus and seizures. Arch Neurol 2002;59:135-7.

Bosnyakova D, Gabova A, Zharikova A, Gnezditski V, Kuznetsova G, van Luijtelaar G. Some peculiarities of time-frequency dynamics of spike-wave discharges in humans and rats. Clin Neurophysiol 2007;118:1736-43.

Challis RE, Kitney RI. Biomedical signal processing (in four parts). Part 3. The power spectrum and coherence function. Med Biol Eng Comput 1991;29:225-41.

Chávez M, Martinerie J, Le Van Quyen M. Statistical assessment of nonlinear causality: application to epileptic EEG signals. J Neurosci Methods 2003;124:113-28.

Coenen AML, van Luijtelaar ELJM. Genetic animal models for absence epilepsy: a review of the WAG/Rij strain of rats. Behav Genet 2003;33: 635-55.

Crick F, Koch C. Constraints on cortical and thalamic projections: the no-strongloops hypothesis. Nature 1998;391(6664):245-50.

Dikanev T, Smirnov D, Wennberg R, Perez Velazquez JL, Bezruchko B. EEG nonstationarity during intracranially recorded seizures: statistical and dynamical analysis. Clin Neurophysiol 2005;116:1796-807.

Feldmann U, Bhattacharya J. Predictability improvement as an asymmetrical measure of interdependence in bivariate time series. Int $\mathrm{J}$ Bifurc Chaos 2004; 14:505-14.

Granger CWJ. Investigating causal relations by econometric models and crossspectral methods. Econometrica 1969;37:424-38.

Granger CWJ, Newbold P. Forecasting economic time series. New York: Academic Press; 1977. p. 333.

Hlavackova-Schindler K, Palus M, Vejmelka M, Bhattacharya J. Causality detection based on information-theoretic approaches in time series analysis. Phys Rep 2007;441:1-46.

Huguenard JR, McCormick DA. Thalamic synchrony and dynamic regulation of global forebrain oscillations. Trends Neurosci 2007;30:350-6.

Inoue M, Duysens J, Vossen JM, Coenen AM. Thalamic multiple-unit activity underlying spike-wave discharges in anesthetized rats. Brain Res 1993;612:35-40.

Jones EG. The thalamus. New York: Plenium Press; 1985. p. 955.

Kaminski M, Ding M, Truccolo WA, Bressler SL. Evaluating causal relations in neural systems: granger causality, directed transfer function and statistical assessment of significance. Biol Cybern 2001;85:145-57. 
Kaplan AYA. Nonstationarity of EEG: methodological and experimental analysis. Usp Fiziol Nauk 1998;29(3):35-55.

Kolb B. Organization of the neocortex in rat. In: Kolb B, Tees R, editors. The cerebral cortex of the rat. Cambridge, Massachusetts: The MIT Press; 1990. p. 21-33.

Le Van Quyen M, Martinerie J, Adam C, Varela F. Nonlinear analyses of interictal EEG map the brain interdependences in human focal epilepsy. Physica D 1999;127:250-66.

Le Van Quyen M, Bragin A. Analysis of dynamic brain oscillations: methodological advances. Trends Neurosci 2007;30:365-73.

Meeren HK, Pijn JP, van Luijtelaar EL, Coenen AM, Lopes da Silva FH. Cortical focus drives widespread corticothalamic networks during spontaneous absence seizures in rats. J Neurosci 2002;22:1480-95.

Meeren H, van Luijtelaar G, Lopes da Silva F, Coenen A. Evolving concepts on the pathophysiology of absence seizures: the cortical focus theory. Arch Neurol 2005;62:371-6.

Midzianovskaia IS, Kuznetsova GD, Coenen AM, Spiridonov AM, van Luijtelaar EL. Electrophysiological and pharmacological characteristics of two types of spike-wave discharges in WAG/Rij rats. Brain Res 2001;911:62-70.

Mormann F, Andrzejak RG, Elger CE, Lehnertz K. Seizure prediction: the long and winding road. Brain 2007;130(Pt 2):314-33.

Panayiotopoulos CP. Absence epilepsies. In: Engel JJ, Pedley TA, editors. Epilepsy: a comprehensive textbook. Philadelphia: Lippincott-Raven Publishers; 1997. p. 2327-46.

Paxinos G, Watson C. The rat brain in stereotaxic coordinates. 2nd ed. New York: Academic Press; 1986.

Pereda E, Quian Quiroga R, Bhattacharya J. Nonlinear multivariate analysis of neurophysiological signals. Prog Neurobiol 2005;77:1-37.
Polack PO, Guillemain I, Hu E, Deransart C, Depaulis A, Charpier S. Deep layer somatosensory cortical neurons initiate spike-and-wave discharges in a genetic model of absence seizures. J Neurosci 2007;27: 6590-9.

Schiff SJ, So P, Chang T, Burke RE, Sauer T. Detecting dynamical interdependence and generalized synchrony through mutual prediction in a neural ensemble. Phys Rev E 1996;54:6708-24.

Seidenbecher T, Staak R, Pape HC. Relations between cortical and thalamic cellular activities during absence seizures in rats. Eur $\mathrm{J}$ Neurosci 1998;10:1103-12.

Sitnikova E, van Luijtelaar G. Cortical and thalamic coherence during spikewave seizures in WAG/Rij rats. Epilepsy Res 2006;71:159-80.

Sitnikova E, van Luijtelaar G. Electroencephalographic characterization of spike-wave discharges in cortex and thalamus in WAG/Rij rats. Epilepsia 2007;48:2296-311, doi:10.1111/j.1528-1167.2007.01250.x.

Steriade M, Amzica F. Dynamic coupling among neocortical neurons during evoked and spontaneous spike-wave seizure activity. J Neurophysiol 1994;72:2051-69.

Steriade M. Sleep, epilepsy and thalamic reticular inhibitory neurons. Trends Neurosci 2005;28:317-24.

van Luijtelaar ELJM, Coenen AML. Two types of electrocortical paroxysms in an inbred strain of rats. Neurosci Lett 1986;70:393-7.

van Luijtelaar G, Sitnikova E. Global and focal aspects of absence epilepsy: the contribution of genetic models. Neurosci Biobehav Rev 2006;30:9831003.

Vergnes M, Marescaux C, Depaulis A, Micheletti G, Warter JM. Spontaneous spike and wave discharges in thalamus and cortex in a rat model of genetic petit mal-like seizures. Exp Neurol 1987;96:127-36. 